\title{
Acute Pneumonia and Importance of Atypical Bacteria
}

I. Boyadjiev, M. Léone, and C. Martin

\section{Introduction}

The term and concept of atypical pneumonia appeared in the 1940s following observations of penicillin-resistant pneumonia [1]. Despite the identification of a large number of microorganisms, the challenge of isolating so-called 'atypical' bacteria is the principal cause of failure of the etiologic diagnosis of pneumonia. These pathogenic agents in the tracheobronchial tree include a large variety of bacteria, viruses

Table 1. Advantages and disadvantages of the different microbiologic diagnostic examinations

\begin{tabular}{|c|c|c|}
\hline Examination & Advantages & Disadvantages \\
\hline Chest x-ray & Distinction pneumonia/bronchitis & Cost \\
\hline Blood culture & Good specificity for S. pneumoniae & Poor sensitivity \\
\hline Direct Gram stain & $\begin{array}{l}\text { Rapidity; isolation of the } \\
\text { predominant pathogen }\end{array}$ & $\begin{array}{l}\text { Does not provide the type of } \\
\text { microorganism }\end{array}$ \\
\hline Cell culture & $\begin{array}{l}\text { Specificity; isolation and } \\
\text { identification of the strain; } \\
\text { antibiotic profile }\end{array}$ & 24 to 72 hours, variable sensitivity \\
\hline $\begin{array}{l}\text { Antigen tests } \\
\text { - immunofluorescence detection } \\
\text { - ELISA and related }\end{array}$ & $\begin{array}{l}\text { Rapidity, very good specificity } \\
\text { automation }\end{array}$ & $\begin{array}{l}\text { Subjective reading, } \\
\text { Sensitivity }=80 \% \\
\text { Specificity }=95 \% \\
\text { Confirmation test necessary }\end{array}$ \\
\hline $\begin{array}{l}\text { Antigen detection } \\
\text { Urinary Legionella }\end{array}$ & Very good specificity & Specific to Legionella pneumophila \\
\hline $\begin{array}{l}\text { Antigen detection } \\
\text { Urinary Histoplasma }\end{array}$ & Very good specificity & Rare pathogen \\
\hline $\begin{array}{l}\text { Molecular tests } \\
\text { Simple hybridization PCR }\end{array}$ & $\begin{array}{l}\text { Automation, sensitivity and } \\
\text { specificity }\end{array}$ & $\begin{array}{l}\text { Sensitivity and specificity=ELISA } \\
\text { Sensitivity to enzyme inhibitors } \\
\text { (false negatives) } \\
\text { Contamination problems, } \\
\text { Cumbersome technique }\end{array}$ \\
\hline Suicide PCR & $\begin{array}{l}\text { Very good sensitivity, } \\
\text { specificity }=100 \%\end{array}$ & Cost, Cumbersome technique \\
\hline
\end{tabular}


and even protozoa. Among atypical bacteria, Chlamydia pneumoniae, Mycoplasma pneumoniae, Legionella pneumoniae, Bordetella pertussis, and Coxiella burnetii are the most widespread. Numerous other bacteria are emerging pathogenic species whose virulence is currently being evaluated. Clinical examination only provides a diagnostic orientation in a restricted number of cases. The availability of rapid and specific microbiologic examination improves the diagnostic performance for this type of pneumonia (Table 1) [2]. Since most of these bacteria are intracellular, diagnosis is based principally on serology.

\section{Microbiologic Techniques for Specific Diagnosis}

\section{Sampling}

The contribution of bronchoalveolar lavage (BAL) in the diagnosis of pulmonary infection has been clearly demonstrated [3]. However, this technique is reserved for the diagnosis of pneumonia in ventilated patients.

\section{Direct Diagnosis}

Direct Examination. Gram-staining has not been assessed for pneumonia caused by atypical bacteria. No direct technique is adequate for direct examination of strict or facultative Gram-negative intracellular bacteria. The weakness of this examination is its very low sensitivity and specificity. Moreover, there is no kit available to detect atypical bacteria, nor those bacteria whose pathogenic roles are beginning to be understood.

Sample Culture. Culturing the pathogenic bacteria remains the method of choice since it provides a microbiologic diagnosis with certainty. However, its relevance depends on the nature of the samples (tracheal aspiration, BAL, pleural liquid, lung biopsy) and the moment they are harvested (immediately following the onset of symptoms and before any antibiotic). Since its isolation is difficult, an atypical bacteria requires specific isolation media. Legionella require BCYE agar, which is sometimes made selective by the addition of antibiotics and/or antifungals. If these antibiotics are ineffective on common bacteria, the samples are treated by acidification $(\mathrm{pH} \mathrm{2})$ and thermal shock before being cultured. The specificity of a culture performed in this manner is $100 \%$ and its sensitivity ranges from 50 to $80 \%$. However, the results are not available before two to three days, and they can be delayed until two to three weeks for some other organisms such as M. pneumoniae. Identification and differentiation of colonies depends on their cultural, biochemical, and enzymatic characteristics as well as their antigenic characteristics in direct immunofluorescence or by agglutination with specific immunserums. The association of these different elements is sometimes not sufficiently discriminating to provide identification of the species. Molecular biology is an innovative tool [4]. Identification of the etiologic agent is particularly important in the perspective of an epidemiologic investigation.

Examination of Samples by Direct Immunofluorescence. Observation by direct immunofluorescence with polyvalent or monovalent conjugates provides a rapid diagnosis. Strict or facultative Gram-negative intracellular bacteria appear as small bacilli. 
The sensitivity of direct immunofluorescence is still unknown and varies from 25 to $70 \%$ [5]. Although there are cross reactions, its specificity is approximately $95 \%$.

Detection of Soluble Antigens. Another method for the rapid diagnosis of atypical pneumonia is the detection of soluble antigens in urine, like for L. pneumophila serogroup 1. The immunoenzymatic (enzyme-like immunosorbent assay [ELISA]) or radioimmunologic methods use polyclonal antibodies. The sensitivity of this test for the detection of $L$. pneumophila serogroup 1 ranges from 60 to $80 \%$ with a $100 \%$ specificity $[6,7]$. On the other hand, there is no test that can detect all of the L. pneumophila serogroups or all Legionella species.

\section{Indirect Diagnosis}

Serologic Diagnosis. Indirect immunofluorescence is still the method of reference. While it is not useful in the acute phase, indirect immunofluorescence provides a retrospective diagnosis. A serum sequence is essential to record the increase in antibodies. This usually appears one week after the beginning of the illness, but can sometimes be much later ( 1 to 9 weeks). A variation of two dilutions between early serum and late serum with a count of $\geq 1 / 128$ for the late serum is sufficient for a diagnosis in the case of L. pneumophila serogroup 1 antibodies. Likewise, the association for $M$. pneumoniae of an antibody titer of $\geq 1 / 64$ and cold agglutinins of $\geq 1 / 64$ provides a diagnosis. According to the literature, the sensitivity of indirect immunofluorescence ranges from 67 to $90 \%$ [8]. Numerous crossed reactions have been described with different species (mycobacteria, leptospires, Chlamydia, Mycoplasma, Citrobacter, Campylobacter, Coxiella burnetii) [8] as well as between the different serogroups and species of Legionella [8]. However, the specificity of immunofluorescence remains good since it ranges from 75 to $99 \%$ [8].

\section{Atypical Bacteria with Known Pathogenic Properties}

\section{Mycoplasma pneumoniae and Chlamydia pneumoniae}

Mycoplasmas are bacteria without walls. This characteristic gives them an extreme plasticity and fragility in an external medium. They are resistant to beta-lactams and their size (inferior to $300 \mathrm{~nm}$ ) allows them to go through antibacterial filters.

The prevalence of $M$. pneumoniae pneumonia in adults ranges from 1.9 to $30 \%$ in the form of necrotizing pneumonia or pleural effusions [9]. Beginning in 1938, a series of cases described atypical pneumonia as characterized by pneumonia with a prolonged duration, no reaction to treatment with penicillin, and non-specific radiological images [10]. The chest X-ray shows either a non-systematized alveolar syndrome or systematized opacities. The inferior lobes are affected more often than the superior. The patient's immunological condition plays a role in the acquisition of a M. pneumoniae pneumonia. Thus, an examination of the BAL fluid of a group of immunosuppressed patients with pleural effusions in the ICU led to the isolation of this bacterium in 5\% of the cases [11]. A serologic examination of two sera sampled at a 10-to-14-day interval is the most common diagnostic method used. However, the diagnosis can be hampered by cross reactions between M. pneumoniae and other commensal mycoplasms among the oropharyngeal florae. There are different opinions on this issue in the literature: a cause of error for some authors 
[12], a negligible parameter for others [13]. At present, the IgA ELISA tests have a sensitivity of 86 to $100 \%$ and a specificity of $100 \%$ for the serologic diagnosis of acute $M$. pneumoniae infection [14].

Chlamydiae are limited by a cytoplasmic membrane and a wall that is comparable to that of Gram-negative bacteria, made up of both an internal and external membrane containing lipopolysaccharides (LPSs) and protein-linked penicillins (PLPs) [15]. They are strict intracellular organisms whose development cycle can be divided into several stages (Fig. 1).

Under certain conditions, the development cycle is altered. There is a delay in the maturation of the reticulate body and an inhibition of infectious elementary body differentiation which is expressed by a persistence of aberrant forms in the host cell. The bacterium is viable but not cultivatable. A parallel can be made with viral latency. C. pneumonia is transmitted through the air. It is responsible for both upper and lower respiratory infections but no symptom is specific. The radiological findings are minor but show extensive bilateral pneumonia with, sometimes, pleural effusion. In adults with chronic bronchitis, immunosuppressed patients, or the elderly, C. pneumoniae can cause severe infections. However, the pathogenic role of this bacteria is now challenged [16].

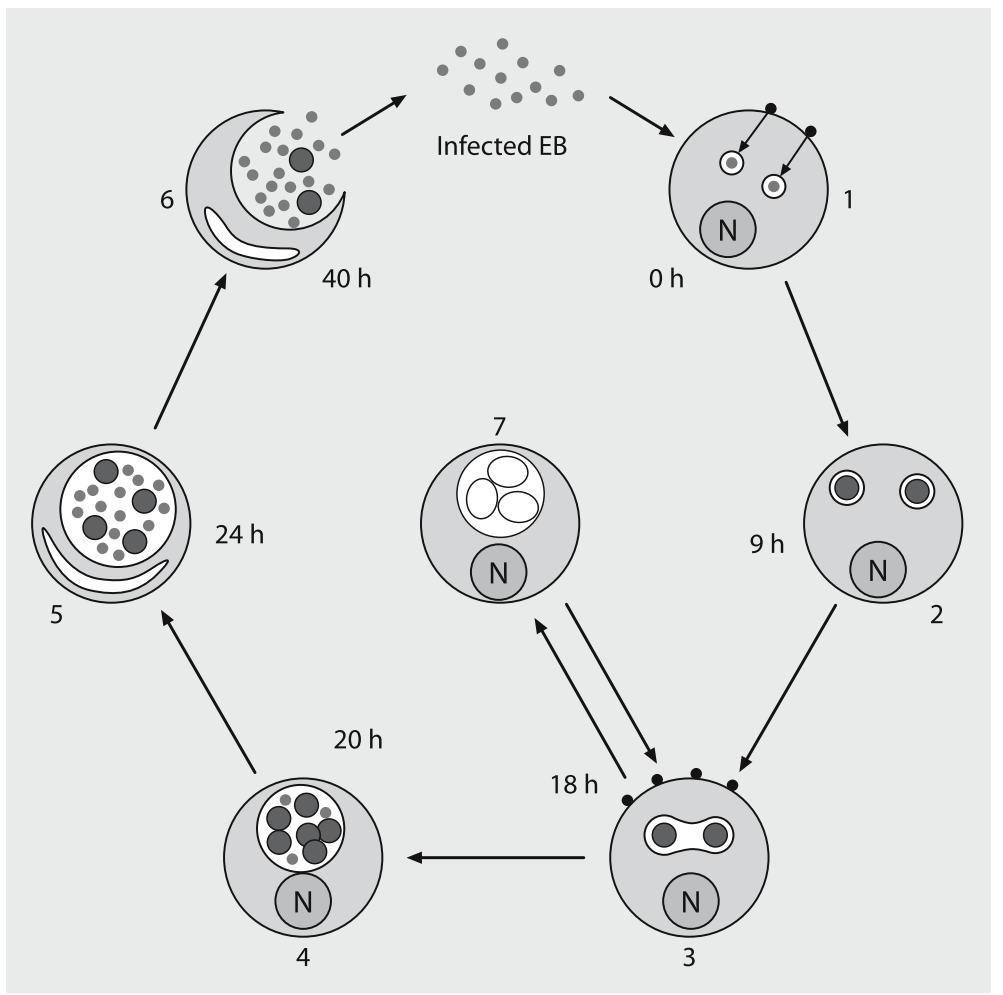

Fig. 1. Development cycle of Chlamydia [21].

1 Attachment and ingestion of elementary bodies (EB) in a vacuole; 2 transformation of EB into reticulated bodies (RB); $\mathbf{3}$ fusion of vacuoles; $\mathbf{4}$ appearance of neoformed EB; 5 maturation of EB; $\mathbf{6}$ cell lysis; productive infection; 7 aberrant bodies: persistent infection, antibiotics, nutriment depletion, interferon- $\gamma$ 
Rapid and precise diagnostic tests for $M$. pneumoniae and C. pneumoniae are not currently available: culture isolation of these bacteria requires the collection of cells by scraping the mucous membranes, then rapid transport at a stable temperature $\left(+4^{\circ} \mathrm{C}\right)$. Amplification techniques have been improved [17]. The most studied and widely used is the polymerase chain reaction (PCR) [18]. Values superior to $98 \%$ have been described in terms of specificity. On the other hand, these tests do not improve sensitivity vis-à-vis culture. Serodiagnosis of $M$. pneumoniae and $C$. pneumoniae is difficult to interpret because seroprevalence is high in the population. Approximately $75 \%$ of subjects have anti-C. pneumoniae antibodies and there are cross reactions with $C$. trachomatis and C. psittasi in 50\% of the cases [19]. Thus, when the etiologic and epidemiologic data and the seroconversion threshold are available, they help in the careful interpretation of the microbiologic results [20].

\section{Coxiella burnetii}

Coxiella burnetii is a strict intracellular microorganism with a wall that is similar to Gram-negative bacteria but with particular properties. C. burnetii proliferates in low $\mathrm{pH}$ phagolysosomes. It appears in two forms, one of which is a resistant form resembling a spore with considerable virulence. In aerosol form, this bacteria can be pathogenic at a concentration of one colony forming unit (cfu). Contamination is essentially respiratory and its incidence is probably underestimated. A retrospective study that included 22 s496 sera was performed between 1982 and 1990 at the Centre National Français de Référence des Rickettsies (French Rickettsia Reference Center). Serologic observation by indirect immunofluorescence was also performed in 1988 on 924 blood-donor sera. The seroprevalence was $4 \%$. The incidence recorded during this 9-year period was 0.58 per 100000 inhabitants [21]. C. burnetii causes Q fever, a polymorphic disease ( $Q$ from the word 'query') $[22,23]$. Infections in man are essentially contracted through the air and by inhaling aerosols in contact with mammals. Current major epidemics are principally linked with livestock [24, 25]. Dissemination by the wind of infected dust is possible making it difficult to confirm animal contact. Q fever is a disease that is more frequently diagnosed, and more severe, in men than in women [26]. Indeed, the sex ratio of 323 patients admitted to hospitals in France with a diagnosis of Q fever between 1982 and 1990 was 2.5/1 [21]. However, systematic research for antibodies directed against $C$. burnetii in the sera of 942 blood donors in the same country revealed a sex ratio of $1 / 1$. Sex hormones, especially estradiol, are said to play a protective role in the development of the disease [27].

From a clinical standpoint, Q fever is extremely polymorphic - this disease can appear in both chronic and acute forms. Diagnosis can only be made by systematic serology. The mean duration of incubation is 20 days but can reach two months, thereby confusing the epidemiologic data [24]. Schematically, there are three forms of acute Q fever which are shown in Figure 2: self-limited flu-like syndrome, hepatitis, and pneumonia. Pulmonary symptoms were the origin of the description of the disease [28]. Most cases are asymptomatic or benign, characterized by a nonproductive cough, fever, and minimal auscultatory abnormalities. In certain cases, pulmonary symptoms can be severe with major hypoxemia or acute respiratory distress syndrome (ARDS) [29]. Hemoptysis can be observed. Chest X-ray reveals nonspecific images of interstitial pneumonia, or, sometimes, an acute lobular pneumonia. The images initially appear in the hilus and are most often located in the inferior lobes [30]. The duration of symptoms ranges from 10 to 90 days and the 


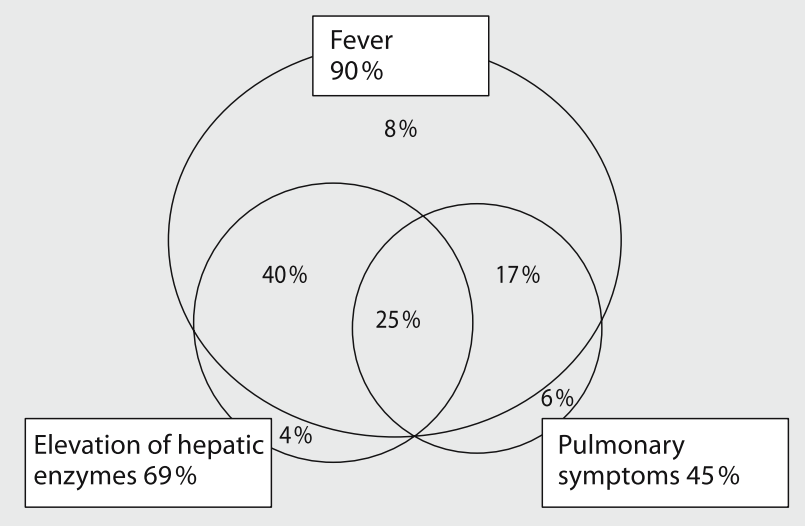

Fig. 2. Relative importance of the three principal clinical forms of $Q$ fever. From [30] with permission

mortality rate is from 0.5 to $1.5 \%$ [21]. Standard biological examinations are nonspecific: the absence of hyperleukocytosis and thrombopenia is of interest. The specific diagnosis is principally based on serology by indirect immunofluorescence.

Treatment requires the use of antibiotics that are active in the specific conditions of growth of this microorganism. Indeed, the proliferation of $C$. burnetii in an acid $\mathrm{pH}$ phagolysosome can explain the potential resistance to antibiotics [31, 32]. Betalactams cannot concentrate in such cells, aminoglycosides are inactive in acid $\mathrm{pH}$ and macrolids are effective in basic $\mathrm{pH}$. Moreover, none of the antibiotics that are active against $C$. burnetii (rifampicin, cotrimoxazole, tetracyclines, clarithromycin and fluoroquinolones) is bactericidal [33]. The prognosis of acute Q fever is usually spontaneously favorable with these bacteriostatic antibiotics [34]. On the other hand, for chronic forms such as endocarditis, bacteriostatic antibiotics control the disease but cannot cure it [35]. Maurin et al. [36] hypothesized that the absence of bactericidal activity of antibiotics against $C$. burnetii was due to their inactivation by the acid $\mathrm{pH}$ in phagolysosomes. By adding a lysosomotropic alkalinizing molecule, hydroxychloroquine, to fluoroquinolones and doxycycline, the authors succeeded in restoring the bactericidal activity of these molecules $[36,37]$.

\section{Legionella and Legionellosis}

Bacteriology. There are 43 different species in the Legionellaceae family including 19 with pathogenic powers. Legionella are facultative intracellular bacteria. After penetrating the cell hosts, they block phagosome activity and phagolysosomal fusion. This mechanism enables the proliferation of Legionella in the phagosome until cellular lysis is triggered.

Legionella are aquatic bacteria that can be found in numerous tanks and freshwater sources that are warm or hot $\left(40\right.$ to $\left.60^{\circ} \mathrm{C}\right)$. Legionella are not often found in cold-water pipes (temperature below $20^{\circ} \mathrm{C}$ ). They are abundant in water-air interfaces such as siphons, ventilators, and especially mixing faucets and shower heads [38]. In buildings, hotels, and hospitals, 60 to $70 \%$ of the samples taken from taps 
are positive [39]. Another essential element in the development of Legionella is their presence in the biofilms of water distribution systems [38]. Finally, the relationships of Legionella with other microorganisms in aquatic media are an essential element of their survival and cast light on the particular epidemiology of legionellosis. The host-parasite relationships with protozoa observed by Rowbotham [40] beginning in 1980 now appear to be fundamental in the pathogenesis and epidemiology of Legionella [41]. Legionella are capable of entering and proliferating in numerous amoebic species. They are found in the phagolysosomal vacuoles according to a process that is in every respect comparable with that observed in infected human macrophages [42]. They survive in amoeba in vitro between 0 and $45^{\circ} \mathrm{C}$. The actively proliferate at $35^{\circ} \mathrm{C}$ in vacuoles and trigger lysis in amoeba cells. The result is bacterial culturing of the environmental medium. Legionella survive also in amoebic cysts. Cysts are extremely resistant entities which protect amoebas when environmental conditions are unfavorable. The Legionella obtained in co-culture preserve their virulence for the experimental subject.

Epidemiology. Legionellosis is a disease linked to our urban civilization. The risk factors are age ( $>50$ years), male, smoker, alcohol, diabetes, and immunosuppression [5]. The mode of transmission is usually inhalation of infectious aerosols due to air conditioning systems, contaminated shower heads, and respiratory device nebulizers and humidifiers [43]. Cases of legionellosis have also been reported after drowning [44] and after the use of bronchoscopes rinsed with tap water [45]. The extrapulmonary mode of transmission for legionellosis (especially endocarditis on a prosthetic valve) is the contamination of surgical wound by infected water [4]. Interhuman contamination has not yet been reported. The incidence and prevalence of legionellosis, including nosocomial cases, are certainly underestimated. Legionella are the origin of 1 to $37 \%$ of hospitalized cases of pneumonia [46]. The incidence of Legionella $(15 \%$ vs $7 \%, p<0.05)$ is significantly larger in intubated patients. Legionella cause 0 to $47 \%$ of nosocomial pneumonia, particularly in immunosuppressed patients [47].

Clinical Signs (Table 2). The clinical signs are not specific to the disease. Legionella is frequently responsible for severe community-acquired pneumonia [46], requiring intensive care. After two to ten days of incubation, patients present with high fever $\left(>39^{\circ} \mathrm{C}\right)$ and bradycardia. In addition, there are symptoms such as watery diarrhea (20 to $40 \%$ of the cases) and neurological signs with confusion. The neurological forms [48] are the signs of encephalic involvement, probably due to a toxin. Cardiac injury is usually located in the pericardium. Endocarditis on valve prostheses has been confirmed by culture [49]. Direct involvement of the digestive tube is in the form of peritonitis or necrotizing colitis. Classic liver lesions are due to the toxin. Renal involvement, observed in 50\% of legionellosis cases, is shown by proteinuria and transitory hematuria. In $13 \%$ of the patients, acute renal failure secondary to rhabdomyolysis can appear. Similarly, signs on chest X ray are observed in $90 \%$ of the cases, and do not distinguish Legionella pneumonia except for their rapid aggravation, especially under inappropriate antibiotics. Nonspecific biological anomalies are observed during systemic involvement. Biological alteration that is more characteristic of legionellosis is hypophosphatemia. However, neither hypophosphatemia, nor hyponatremia, nor elevated transaminases alone constitute a specific diagnostic criterion. Their association with other symptoms could cause one to suspect legionellosis. 
Diagnosis. In its classic form, legionellosis is characterized by a febrile pneumonia, watery bloodless diarrhea and confusion (Table 2). Resistance to treatment by betalactams and bradycardia are also good orientation elements [50]. The existence of five to $10 \%$ mixed infections makes clinical diagnosis difficult and requires a bacteriological diagnosis. Bacteriological diagnosis of legionellosis is difficult. The culture has a specificity of $100 \%$ but a sensitivity of 50 to $80 \%$ after a growth period of at least two or three days. Observation by direct immunofluorescence with conjugates provides a rapid diagnosis - Legionella appears as small bacilli. The sensitivity of this examination is poorly known: 25 to $75 \%$ of the positive cultures have a positive direct immunofluorescence [8]. Detection of the urinary antigens of Legionella pneumophila serogroup 1 is considered as a specific, rapid and confirmed diagnosis. Urinary antigens are detected from the first days of the infection and up to 60 days later, as after appropriate antibiotics [9]. However, serology remains the most frequent means to diagnose legionellosis even though it is often late or even retrospective.

Even though it is a major cause of pneumonia, legionellosis is still underestimated. The combined use of urinary antigen test and cultures improves the diagnosis. PCR is a promising tool but standardized test kits are not available. The development of both antigen urinary tests that detect a larger number of Legionella species and standardized PCR methods could make up this current shortcoming.

The bactericidal antibiotics are rifampicin, macrolides, fluoroquinolones, and aminoglycosides. Antibiotic treatment of legionellosis produced by infected aerosols in experimental subjects has confirmed the excellent in vivo efficacy of these antibiotics [51]. The duration of treatment must be 15 days [51].

Table 2. Frequency (in percentage) of clinical and biological signs during legionellosis [52]

\begin{tabular}{|c|c|c|c|}
\hline \multicolumn{2}{|l|}{ Clinical and physical signs } & \multicolumn{2}{|l|}{ Biological signs } \\
\hline Fever & & & \\
\hline$>39^{\circ} \mathrm{C}$ & 90 & Leukocytes $>10000 / \mathrm{mm}^{3}$ & 45 \\
\hline$>40^{\circ} \mathrm{C}$ & 50 & & \\
\hline Cough & $80-100$ & Hyponatremia & 68 \\
\hline Chills & 75 & & \\
\hline Expectoration & 50 & Hypophosphoremia & 51 \\
\hline Dyspnea & $54-94$ & Increase in enzymes & \\
\hline Thoracic pain & $25-33$ & LDH & 45 \\
\hline Diarrhea & 50 & ASAT & 65 \\
\hline Nausea, vomiting & 25 & Alcaline phosphatase & 62 \\
\hline Abdominal pain & $15-23$ & Hyperbilirubinemia & 15 \\
\hline Cephalgia & $41-71$ & Proteinuria & 50 \\
\hline Confusion & $30-40$ & Hematuria & 50 \\
\hline Myalgia & $14-83$ & & \\
\hline Bradycardia & 60 & & \\
\hline Râles & 80 & & \\
\hline Dullness on percussion & $80-100$ & & \\
\hline
\end{tabular}




\section{- Conclusion}

The diagnosis of pulmonary infection caused by Mycoplasma and Chlamydia pneumoniae, Coxiella burnetii, and different species of Legionella, is often long and challenging although they are the major etiologic agents of pneumonia. For this reason, the treatment of these infections remains probabilistic. Advances in new diagnostic techniques, such as PCR sequencing, show the relative predominance of atypical organisms and serves to identify emerging pathogenic agents. Moreover, these techniques should clarify the correlation between common and atypical pathogens.

\section{References}

1. Eaton M, Meikeljohn G, Van Herick W (1944) Studies on the etiology of primary atypical pneumonia: A filtrable agent transmissible to cotton rats, hamsters and chick embryos. J Exp Med 79:649-661

2. Raoult D, Fournier P, Drancourt M (2004) What does the future hold for clinical microbiology? Nat Rev Microbiol 2:151-159

3. Aubas S, Aubas P, Capdevila X, Darbas H, Roustan J, du Cailar J (1994) Bronchoalveolar lavage for diagnosing bacterial pneumonia in mechanically ventilated patients. Am J Respir Crit Care Med 149:860-866

4. Rosenstraus M, Wang Z, Chang S, DeBonville D, Spadoro J (1998) An internal control for routine diagnostic PCR: design, properties, and effect on clinical performance. J Clin Microbiol 36:191-197

5. Edelstein P (1993) Legionnaires' disease. Clin Infect Dis 16:741-749

6. Harrison T, Uldum S, Alexiou-Daniel S, et al (1998) A multicenter evaluation of the Biotest legionella urinary antigen EIA. Clin Microbiol Infect 4:359-365

7. Birtles R, Harrison T, Samuel D, Taylor A (1990) Evaluation of urinary antigen ELISA for diagnosing Legionella pneumophila serogroup 1 infection. J Clin Pathol: 685-690

8. Bornstein N, Fleurette J (1994) Legionella. In: Freney J, Renaud F, Hansen D, Bollet C (eds) Manuel de Bactériologie Clinique. Elsevier, Paris, pp 1327-1354

9. Hammerschlag M (2001) Mycoplasma pneumoniae infections. Curr Opin Infect Dis 14:181186

10. Reimann H (1984) Landmark article Dec 24,1938: An acute infection of the respiratory tract with atypical pneumonia. A disease entity probably caused by a filtrable virus. By Hobart A. Reimann. JAMA 251:936-944

11. Parides G, Bloom J, Ampel N, Ray C (1988) Mycoplasma and ureaplasma in bronchoalveolar lavage fluids from immunocompromised hosts. Diagn Microbiol Infect Dis 9:55-57

12. Sasaki T, Bonissol C, Stoiljkovic B (1987) Cross-reactive antibodies to mycoplasmas found in human sera by the enzyme-linked immunosorbent assay (ELISA). Microbiol Immunol 31:521-530

13. Bredt W, Kleinmann B, Jacobs E (1987) Antibodies in the sera of Mycoplasma pneumoniaeinfected patients against proteins of Mycoplasma genitalium and other mycoplasmas of man. Zentralbl Bakteriol Mikrobiol Hyg 266:32-42

14. Watkins-Riedel T, Stanek G, Daxboeck F (2001) Comparison of SeroMP IgA with four other commercial assays for serodiagnosis of Mycoplasma pneumoniae pneumonia. Diagn Microbiol Infect Dis 40:21-25

15. Grayston J, Wang S, Kuo C, Campbell L (1989) Current knowledge on Chlamydia pneumoniae, strain TWAR, an important cause of pneumonia and other acute respiratory diseases. Eur J Clin Microbiol Infect Dis 8:191-202

16. Danesh J (2005) Antibiotics in the prevention of heart attacks. Lancet 365:365-367

17. Ramirez J, Ahkee S, Tolentino A, Miller R, Summersgill J (1996) Diagnosis of Legionella pneumophila, Mycoplasma pneumoniae, or Chlamydia pneumoniae lower respiratory infection using the polymerase chain reaction on a single throat swab specimen. Diagn Microbiol Infect Dis 24:7-14 
18. Laudenbach V, Mantz J, Desmonts J (1999) Understanding of molecular biology. Ann Fr Anesth Reanim 18:725-747

19. Orfila J, Chaigneau C, Gommeux A, Sueur J, Fey T (1995) Epidémiologie des infections respiratoires à Chlamydia Pneumoniae dans le département de la Somme. Bull Epidemiol Hebd 42:186-187

20. Marston B, Plouffe J, File T, et al (1997) Incidence of community-acquired pneumonia requiring hospitalization. Results of a population-based active surveillance Study in Ohio. The Community-Based Pneumonia Incidence Study Group. Arch Intern Med 157:1709-1718

21. Tissot Dupont H, Raoult D, Brouqui P, et al (1992) Epidemiologic features and clinical presentation of acute Q fever in hospitalized patients: 323 French cases. Am J Med 93:427-434

22. Mege J, Maurin M, Capo C, Raoult D (1997) Coxiella burnetii: the 'query' fever bacterium. A model of immune subversion by a strictly intracellular microorganism. FEMS Microbiol Rev 19:209-217

23. Raoult D (1996) Q fever: still a query after all these years. J Med Microbiol 44:77-78

24. Dupuis G, Petite J, Peter O, Vouilloz M (1987) An important outbreak of human Q fever in a Swiss Alpine valley. Int J Epidemiol 16:282-287

25. Dupuis G, Peter O, Pedroni D, Petite J (1985) Clinical aspects observed during an epidemic of 415 cases of Q fever. Schweiz Med Wochenschr 115:814-818

26. Maurin M, Raoult D (1999) Q fever. Clin Microbiol Rev 12:518-553

27. Leone M, Honstettre A, Lepidi H, et al (2004) Effect of sex on Coxiella burnetii infection: protective role of 17beta-estradiol. J Infect Dis 189:339-345

28. Derrick E (1937) Q fever, a new fever entity: clinical features, diagnosis and laboratory investigation. Med J Aust 2:281-299

29. Marrie T (1995) Coxiella burnetii (Q fever) pneumonia. Clin Infect Dis Suppl 3:S253-S264

30. Fournier P (1999) Fièvre Q In: Bricaire F (ed) Encycl Med Chir, Maladies Infectieuses, Elsevier, Paris, 8-037-I-10

31. Raoult D, Brouqui P (1993) Intracellular location of microorganisms. In: Raoult D (ed) Antimicrobial Agents and Intracellular Pathogens. CRC Press, Boca Raton, pp 39-62

32. Hackstadt T, Williams J (1981) Biochemical stratagem for obligate parasitism of eukaryotic cells by Coxiella burnetii. Proc Natl Acad Sci USA 78:3240-3244

33. Subramanya N, Wright J, Khan M (1982) Failure of rifampicin and co-trimoxazole in Q fever endocarditis. BMJ 285:343-344

34. Raoult D (1993) Treatment of Q fever. Antimicrob Agents Chemother 37:1733-1736

35. Marrie T, Raoult D (2002) Update on Q fever, including Q fever endocarditis. Curr Clin Top Infect Dis 22:97-124

36. Maurin M, Benoliel A, Bongrand P, Raoult D (1992) Phagolysosomal alkalinization and the bactericidal effect of antibiotics: the Coxiella burnetii paradigm. J Infect Dis 166:1097-1102

37. Raoult D, Drancourt M, Vestris G (1990) Bactericidal effect of doxycycline associated with lysosomotropic agents on Coxiella burnetii in P388D1 cells. Antimicrob Agents Chemother 34:1512-1514

38. Brundrett G (1992) Legionella and Building Services. Butterworth Heinemann, Oxford.

39. Greub G, Boyadjiev I, La Scola B, Raoult D, Martin C (2003) Serological hint suggesting that Parachlamydiaceae are agents of pneumonia in polytraumatized intensive care patients. Ann NY Acad Sci 990:311-319

40. Rowbotham TJ (1980) Preliminary report on the pathogenicity of Legionella pneumophila for freshwater and soil amoebae. J Clin Pathol 33:1179-1183

41. Kwait Y, Gao L, Stone B, Venkataraman C, Harb O (1998) Invasion of Protozoa by Legionella pneumophila and its role in bacterial ecology and pathogenesis. Appl Environ Microbiol 64:3127-3133

42. Joshi A, Swanson M (1999) Comparative analysis of Legionella pneumophila and Legionella micdadei virulence traits. Infect Immun 67:4134-4142

43. Woo A, Goetz A, Yu V (1992) Transmission of Legionella by respiratory equipment and aerosol generating devices. Chest 102:1586-1590

44. Tokuda H, Yahagi N, Kasai S, Kitamura S, Otsuka Y (1997) A case of fatal pneumonia caused by Legionella pneumophila serogroup 6 developed after drowning in a public bath. Kansenshogaku Zasshi 71:169-174 
45. Mitchell D, Hicks L, Chiew R, Montanaro J, Chen S (1997) Pseudoepidemic of Legionella pneumophila serogroup 6 associated with contaminated bronchoscopes. J Hosp Infect 37:19-23

46. Rello J, Bodi M, Mariscal D, Navarro M, Diaz E, Gallego M, Valles J (2003) Microbiological testing and outcome of patients with severe community-acquired pneumonia. Chest 123:174-180

47. Hutchinton O (1990) Nosocomial legionellosis. Rev Med Microbiol 1:108-115

48. Spieker S, Petersen D, Rolfs A, Fehrenbach F, Kuntz R, Seuffe R (1998) Acute disseminated encephalomyelitis following Pontiac fever. Eur Neurol 40:169-172

49. Brouqui P, Raoult D (2001) Endocarditis due to rare and fastidious bacteria. Clin Microbiol Rev 14:177-207

50. Stout J, Yu V (1997) Legionellosis. N Engl J Med 337:682-687

51. Vandenesch F, Surgot M, Bornstein N, Paucod J, Marmet D, Isoard P (1990) Relationship between free Amoeba and Legionella: studies in vitro and in vivo. Zentralbl Bakteriol 272:265-275

52. Sile S, Straub T, Christmann B (1994) Manifestations extra-pulmonaires des légionelloses. Med Mal Infect 24:874-880 